\title{
Reconsideration on the role of the specific heat ratio in Arrhenius law applications
}

\author{
Yunfeng Liu · Zonglin Jiang
}

Received: 21 March 2007 / Revised: 27 November 2007 / Accepted: 30 November 2007 / Published online: 6 March 2008

(C) Springer-Verlag 2008

\begin{abstract}
Arrhenius law implicates that only those molecules which possess the internal energy greater than the activation energy $E_{\text {a }}$ can react. However, the internal energy will not be proportional to the gas temperature if the specific heat ratio $\gamma$ and the gas constant $R$ vary during chemical reaction processes. The varying $\gamma$ may affect significantly the chemical reaction rate calculated with the Arrhenius law under the constant $\gamma$ assumption, which has been widely accepted in detonation and combustion simulations for many years. In this paper, the roles of variable $\gamma$ and $R$ in Arrhenius law applications are reconsidered, and their effects on the chemical reaction rate are demonstrated by simulating onedimensional C-J and two-dimensional cellular detonations. A new overall one-step detonation model with variable $\gamma$ and $R$ is proposed to improve the Arrhenius law. Numerical experiments demonstrate that this improved Arrhenius law works well in predicting detonation phenomena with the numerical results being in good agreement with experimental data.
\end{abstract}

Keywords Arrhenius law - Detonation - Specific heat ratio

\section{Introduction}

Arrhenius in 1889 stated that only molecules possessing energy greater than a certain amount $E_{\mathrm{a}}$ will react, and introduced a Boltzmann factor for the first time to calculate the

\section{Y. Liu (凶) · Z. Jiang}

Key Laboratory of High Temperature Gas Dynamics, Institute of Mechanics, Chinese Academy of Sciences, Beijing 100080, China

e-mail: yfliu@pku.org.cn

Z. Jiang

e-mail: zljiang@imech.ac.cn chemical reaction rate. The expression [1] of

$k=A \exp \left(-\frac{E_{\mathrm{a}}}{R_{\mathrm{u}} T}\right)$

is referred to as the Arrhenius law, where $k$ is the chemical reaction rate constant, $A$ the pre-exponential factor, $E_{\mathrm{a}}$ the activation energy, $R_{\mathrm{u}}$ the universal gas constant, and $T$ the absolute temperature in Kelvin. The factor $A$ is assumed to include the effect of molecule collisions.

For a reacting system with a constant specific ratio $\gamma$ and constant gas constant $R$, the internal energy is proportional only to the temperature. But in the chemical reaction processes where $\gamma$ and $R$ are varying, the internal energy is not necessarily proportional to the temperature, and can be a function of both gas temperature and the specific heat ratio. Therefore, in this case the term $R_{\mathrm{u}} T$ in Eq. (1) does not mean the internal energy though it has energy dimension, and the chemical reaction rate constant $k$ calculated by Eq. (1) will become incorrect, especially at the flame or detonation front.

The overall one-step chemical reaction model with the constant $\gamma$ and $R$ has been widely used to simulate detonation phenomena for many decades and propulsive performance of pulse detonation engines recently [2-4] because of its simplicity. It has been realized that the employment of constant $\gamma$ and $R$ for both reactants and products inevitably introduces errors in thermodynamic variable prediction $[4,5]$. Therefore, in Ref. [6], a formulation for calculating variable $\gamma$ across detonation front was proposed, and it was formulated with the interpolation between the specific heat ratio of the unburned and burned gases to study three-dimensional detonation wave structures. However, it is observed from the numerical results that the pre-exponential factor is dependent on the grid resolution, and has to be tuned according to different grid resolutions; otherwise, the cellular cell structures cannot be well simulated with the proposed model of variable $\gamma$. 
This problem arises not only because the variable $\gamma$ has to apply, but also because the term $R_{\mathrm{u}} T$ in the Arrhenius law must be re-evaluated according to the $\gamma$ variation. In this study, the role of variable $\gamma$ in Arrhenius law applications was reconsidered, and its effect on chemical reaction rate calculations was demonstrated by simulating the one-dimensional $\mathrm{C}-\mathrm{J}$ and two-dimensional cellular detonations. A new overall one-step detonation model with variable $\gamma$ and $R$ was proposed to modify the Arrhenius law expression. Some numerical tests were carried out to demonstrate the performance of this new detonation model.

\section{Governing equations}

The governing equations for the present numerical study are chosen to be the two-dimensional conservation equations of mass, momentum and energy, implemented with finiterate chemical reaction kinetics. Viscous terms and diffusive effects are neglected because of their minor roles in determining the overall flow dynamics of detonation. The chemical reaction is modeled by using a simplified one-step irreversible kinetics model. The governing equations read

$\frac{\partial \boldsymbol{U}}{\partial t}+\frac{\partial \boldsymbol{F}}{\partial x}+\frac{\partial \boldsymbol{G}}{\partial y}=\boldsymbol{S}$,

$\boldsymbol{U}=\left(\begin{array}{c}\rho \\ \rho u \\ \rho v \\ \rho e \\ \rho Z\end{array}\right), \quad \boldsymbol{F}=\left(\begin{array}{c}\rho u \\ \rho u^{2}+p \\ \rho u v \\ (\rho e+p) u \\ \rho u Z\end{array}\right)$,

$\boldsymbol{G}=\left(\begin{array}{c}\rho v \\ \rho u v \\ \rho v^{2}+p \\ (\rho e+p) v \\ \rho v Z\end{array}\right), \quad \boldsymbol{S}=\left(\begin{array}{c}0 \\ 0 \\ 0 \\ 0 \\ \dot{\omega}\end{array}\right)$,

where, $\rho, u, v, e$, and $Z$ represent the density, axial and lateral velocity components, specific total energy, and the reaction progress variable (i.e., mass fraction of reactants), respectively. The pressure $p$ is calculated with the equation of state,

$p=(\gamma-1) \rho\left[e-\left(u^{2}+v^{2}\right) / 2-Z q\right]$,

where, $\gamma$ is the specific heat ratio and $q$ is the heat released per unit mass of reactants. For a one-step irreversible reaction model, the mass production rate of products $\dot{\omega}$ is defined as

$\dot{\omega}=-K \rho Z \exp \left(-E_{\mathrm{a}} / R T\right)$,

where, $K$ is the pre-exponential factor, $T$ the temperature, $E_{\mathrm{a}}$ the activation energy per unit mass of reactants, and $R$ the gas constant. In this model, $\gamma$ and $R$ are assumed to be the same constants both for reactants and products. This model has been widely applied for many years because of its simplicity.

The six parameters involved in Eqs. (4) and (5) for stoichiometric hydrogen/air system are chosen to be [4]

$$
\begin{aligned}
& Z=1.0, \\
& \gamma=1.290, \\
& R=368.9 \mathrm{~J} /(\mathrm{kg} \mathrm{K}), \\
& q=2.720 \times 10^{6} \mathrm{~J} / \mathrm{kg}, \\
& E_{\mathrm{a}}=4.794 \times 10^{6} \mathrm{~J} / \mathrm{kg}, \\
& K=7.5 \times 10^{9} \mathrm{~s}^{-1} .
\end{aligned}
$$

By considering the problem arising from the constant assumption of $\gamma$ and $R$, a variable $\gamma(Z)$ was introduced in Ref. [6] which takes the following expression

$$
\gamma(Z)=\frac{\gamma_{\mathrm{U}}\left(\gamma_{\mathrm{B}}-1\right) Z+\gamma_{\mathrm{B}}\left(\gamma_{\mathrm{U}}-1\right)(1-Z)}{\left(\gamma_{\mathrm{B}}-1\right) Z+\left(\gamma_{\mathrm{U}}-1\right)(1-Z)},
$$

where, $\gamma_{U}$ and $\gamma_{B}$ are the specific heat ratio for reactants and products, respectively. Since non-dimensional governing equations were used, the gas constant $R$ did not appear, therefore, was not discussed in Ref. [6].

Considering the dimensional governing equations and variable gas constant $R$, we introduce a new variable gas constant $R(Z)$ in this study, and modify Eq. (7) into the following equations

$$
\begin{aligned}
& \gamma(Z)=\frac{\gamma_{\mathrm{U}} R_{\mathrm{U}} Z /\left(\gamma_{\mathrm{U}}-1\right)+\gamma_{\mathrm{B}} R_{\mathrm{B}}(1-Z) /\left(\gamma_{\mathrm{B}}-1\right)}{R_{\mathrm{U}} Z /\left(\gamma_{\mathrm{U}}-1\right)+R_{\mathrm{B}}(1-Z) /\left(\gamma_{\mathrm{B}}-1\right)}, \\
& R(Z)=R_{\mathrm{U}} Z+R_{\mathrm{B}}(1-Z),
\end{aligned}
$$

where, $R_{\mathrm{U}}$ and $R_{\mathrm{B}}$ are the gas constants for reactants and products, respectively. From Eqs. (8) and (9), it can bee seen that the variables $\gamma(Z)$ and $R(Z)$ depend on only the reaction progress variable $Z$, which agrees with our common understanding.

For the newly proposed model, the constant parameters for the stoichiometric hydrogen/air system are taken to be [5]

$$
\begin{aligned}
& Z_{\mathrm{U}}=1.0, \\
& Z_{\mathrm{B}}=0.0, \\
& \gamma_{\mathrm{U}}=1.40, \\
& \gamma_{\mathrm{B}}=1.24, \\
& R_{\mathrm{U}}=398.5 \mathrm{~J} /(\mathrm{kg} \mathrm{K}), \\
& R_{\mathrm{B}}=368.9 \mathrm{~J} /(\mathrm{kg} \mathrm{K}), \\
& q=3.50 \times 10^{6} \mathrm{~J} / \mathrm{kg}, \\
& E_{\mathrm{a}}=4.794 \times 10^{6} \mathrm{~J} / \mathrm{kg}, \\
& K=7.5 \times 10^{9} \mathrm{~s}^{-1},
\end{aligned}
$$

where, $\gamma_{\mathrm{U}}, \gamma_{\mathrm{B}}, R_{\mathrm{U}}$, and $R_{\mathrm{B}}$ are determined from the detailed chemical reaction kinetics [5,7]. The specific heat release $q$ is calculated from the following simplified equation [8]

$$
D_{\mathrm{CJ}}=\sqrt{2\left(\gamma_{\mathrm{B}}^{2}-1\right) q},
$$


where, $D_{\mathrm{CJ}}$ is the Chapman-Jouguet detonation speed. For the stoichiometric hydrogen/air system, the detonation speed is $D_{\mathrm{CJ}}=1,980 \mathrm{~m} / \mathrm{s}$.

In order to study the role of the variable specific heat ratio in the Arrhenius law applications, the activation energy $E_{\mathrm{a}}$ in Eq. (5) should be modified by taking into account the influence of the specific heat ratio on the internal energy. The new equation can be written as:

$\dot{\omega}=-K \rho Z \exp \left(-E_{\mathrm{a}}^{*}(\gamma(Z)-1) / R(Z) T\right)$,

$E_{\mathrm{a}}^{*}=E_{\mathrm{a}} /\left(\gamma_{\mathrm{B}}-1\right)$,

where, the term $R(Z) T /(\gamma(Z)-1)$ represents the averaged internal energy.

In the following numerical simulations, the equations from Eqs. (2)-(6) are the first detonation model, and referred to as model-A for the sake of simplicity. The equations of the second model are Eqs. (2)-(5) and (8)-(10), and are called model-B. In these two models, the activation energy in the Arrhenius law is actually the activation temperature, as widely applied. The equations for model-C are Eqs. (2)-(4), (8)-(10), and (12)-(13), in which the activation energy is used instead of the activation temperature.

\section{Numerical methods and setup}

The convective terms of the governing equations are evaluated by using three-order ENO scheme [9]. Steger-Warming flux splitting method is employed to account for upwind effect [10]. The time integration is completed with the thirdorder TVD Runge-Kutta method [9]. The reflection boundary conditions are applied on solid walls. All the initial and boundary conditions are the same for all the simulation cases with three detonation models.

In one-dimensional simulations, the computational domain is a straight detonation tube with the left end closed and the right end open. The tube is fully filled with the premixed stoichiometric hydrogen/air mixture at 1 atm and $300 \mathrm{~K}$. A small initiation region with high pressure and high temperature reactants is specified near the closed end. The detonation tube is $50 \mathrm{~mm}$ long, and the grid spacing is $\mathrm{d} x=10 \mu \mathrm{m}$. This one-dimensional problem is used to quantitatively compare thermodynamic properties calculated with three detonation models.

In two-dimensional numerical simulations, the computational domain is a two-dimensional straight channel which moves together with the detonation front. The reactants enter the computational domain from its right, and the products leave from its left. The left boundary is extrapolated under the uniform flow assumption. With the proper choice of the reference frame speed, the detonation front eventually sits near the right boundary. A small initiation region with high pressure and high temperature reactants is specified near the left boundary. It will produce an overdriven detonation that naturally attenuates to the $\mathrm{C}$-J steady state. The detonable mixture is also the premixed stoichiometric hydrogen/air at 1 atm and $300 \mathrm{~K}$. The width of the computational domain is 4, 2, and $8 \mathrm{~mm}$ for model-A, B, and $\mathrm{C}$, respectively. The ratio of length to width is 6 to minimize the influence of the outflow boundary conditions. The equally spaced grids are $\mathrm{d} x=\mathrm{d} y=10 \mu \mathrm{m}$. This test case is selected to investigate the chemical reaction kinetics predicted with three detonation models by examining the cellular cell patterns because the cellular detonation is strongly dependant on chemical reaction models.

\section{Results and discussion}

\subsection{Thermodynamic properties}

Figure 1 shows pressure profiles of the one-dimensional detonations simulated with three reaction models at different instants. For the test case with stoichiometric hydrogen/air mixture at $1 \mathrm{~atm}$ and $300 \mathrm{~K}$, it was known from theoretical analysis [8] that the constant pressure at the closed end is $5.6 \mathrm{~atm}$, the post-shock pressure (von Neumann spike) is $26.5 \mathrm{~atm}$, and the detonation speed $D_{\mathrm{CJ}}=1,980 \mathrm{~m} / \mathrm{s}$. From these pressure profiles, it can be seen that both model-B and model-C predict the closed end pressure well, while modelA predicts a value of $5.8 \mathrm{~atm}$, being 0.2 atm higher than the theoretical result. On the other hand, both model-A and model-C give a reasonable value of the von Neumann spike pressure, but it was much under-estimated by model-B. So, only model-C predicts both parameters well, and it will be

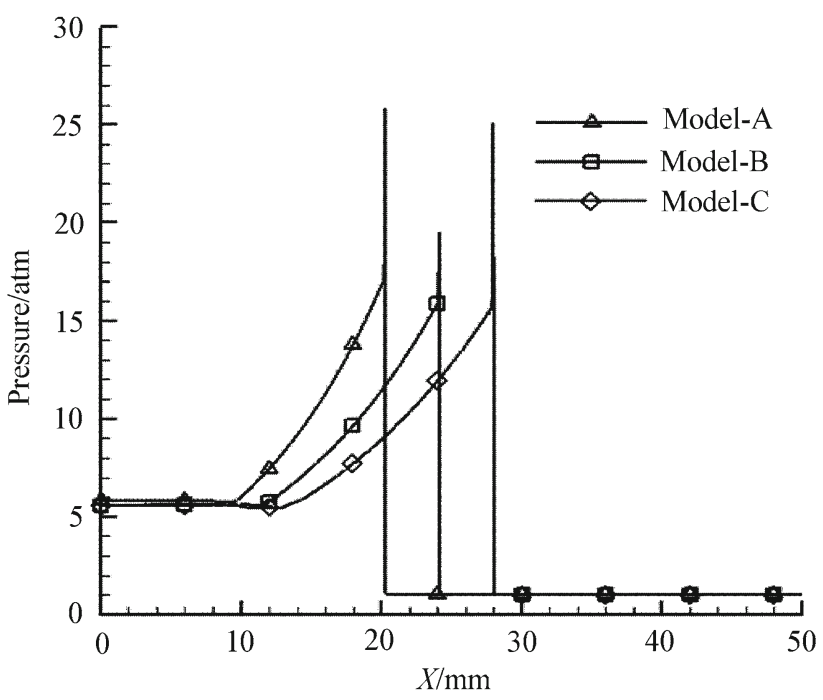

Fig. 1 Pressure profiles predicted with three detonation models at different instants 


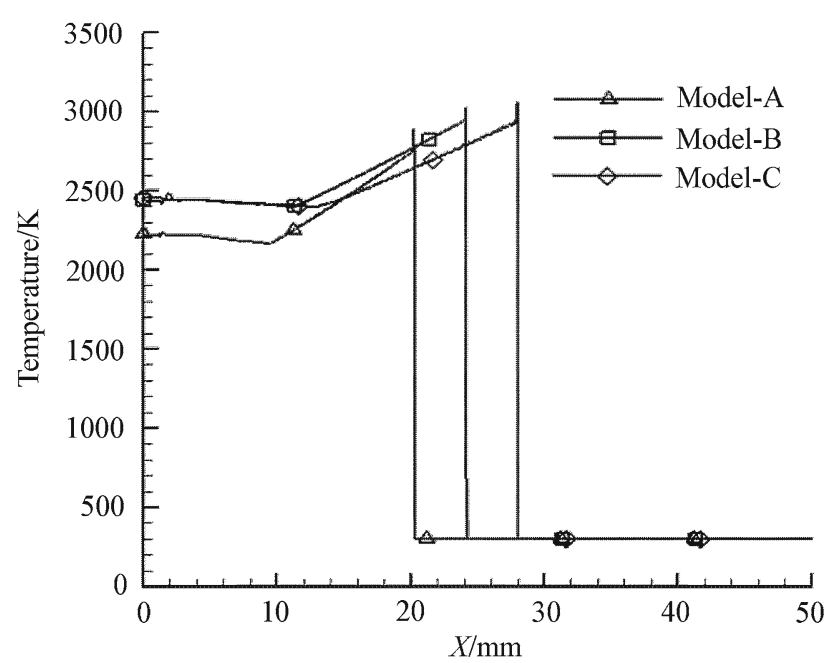

Fig. 2 Temperature profiles predicted with three models at different instants

found in the following two-dimensional simulations that this property of model-B will influence its capability to simulate the cellular structure of detonation fronts.

Temperature profiles from the same numerical results are presented in Fig. 2. The temperature profiles calculated with model-B and model-C are almost the same, but the one predicted by model-A is $200 \mathrm{~K}$ lower. This result implies that the thermodynamic properties can be under-predicted with model-A with the constant $\gamma$ assumption. However, these three detonation models almost give the same detonation speed of $D_{\mathrm{CJ}}=1,980 \mathrm{~m} / \mathrm{s}$ because the detonation speed is dominated by Eq. (11), and depends on the total heat release.

By summarizing the comparison of the one-dimensional simulations, it can be concluded that only model-C works well in predicting the closed end pressure, the post-shock pressure, and the detonation temperature.

\subsection{Chemical reaction kinetic properties}

Figure 3 shows the pressure and temperature contours of two-dimensional cellular detonation simulated with modelA. The cell pattern evolution during numerical simulations is presented in Fig. 4. There are about six detonation cells observable in this $4 \mathrm{~mm}$ wide channel after the detonation gets stable. The averaged width of the detonation cell is about $0.67 \mathrm{~mm}$. If a disturbance is placed in front of the detonation front, the detonation front is disturbed, but it reaches stable states quickly, and the cell width becomes about $0.8 \mathrm{~mm}$. The cell width measured in experiments is about $8.4 \mathrm{~mm}$ for the stoichiometric hydrogen/air mixture at $1 \mathrm{~atm}$ and $300 \mathrm{~K}$ according to Ref. [11], which is about ten times as big as this numerical size. It is clear that the detonation cell size is much under-estimated with model-A.

Pressure and temperature contours of two-dimensional cellular detonations simulated by model-B are shown in Fig. 5. From this figure, it is observable that there are six pairs of triple-wave points in this $2 \mathrm{~mm}$ wide channel, and the width of the cell is about $0.3 \mathrm{~mm}$, which is too small to be physically correct.

The cellular cell pattern evolution during numerical simulations is presented in Fig. 6. Even though an obstacle is placed in front of the detonation front, the small cell pattern is quickly recovered after the detonation front propagates over the obstacle. It means that the small cell size has nothing to do with the initial disturbances and boundary conditions, but depends mainly on the chemical reaction kinetics simulated with model-B.
Fig. 3 Pressure and temperature contours of two-dimensional cellular detonation simulated with model-A. a Pressure; b temperature

Fig. 4 Cell pattern evolution during simulations calculated with model-A
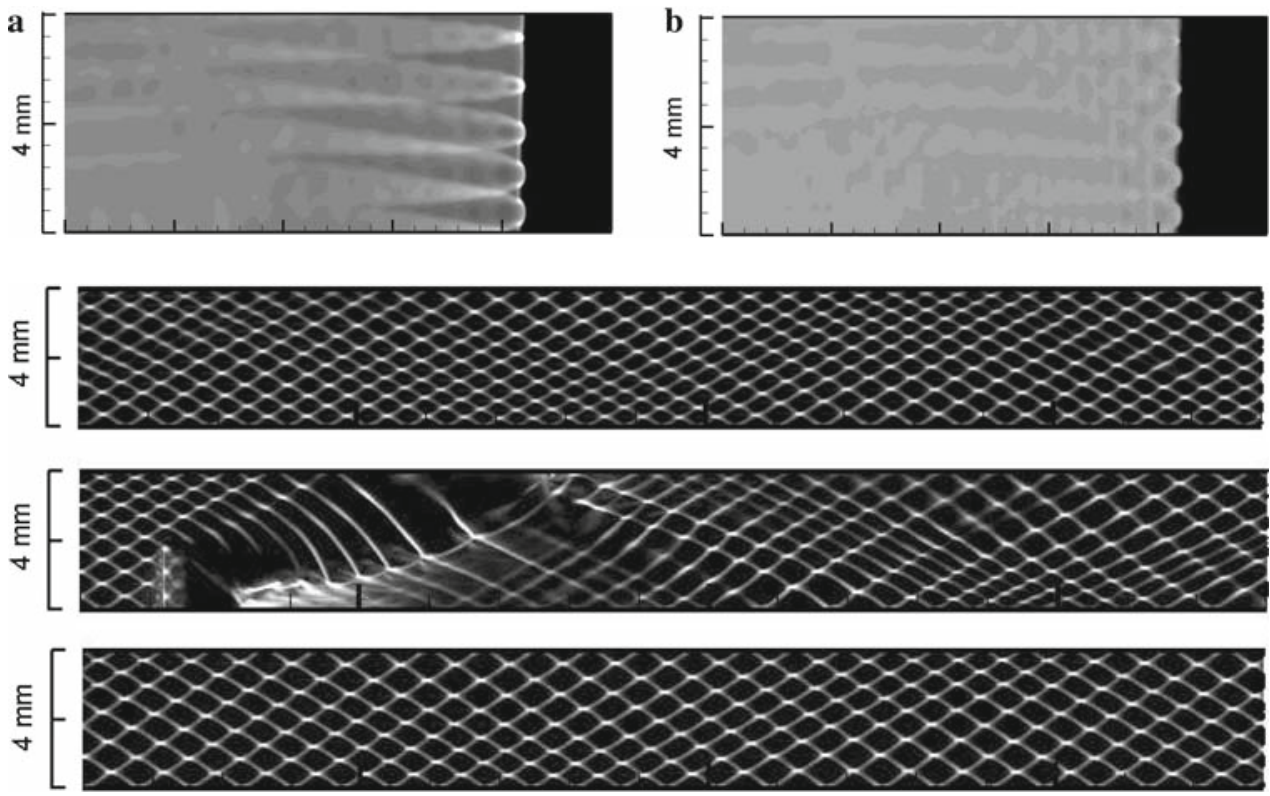
Fig. 5 Pressure and temperature contours of two-dimensional cellular detonation simulated with model-B. a Pressure;

b temperature

Fig. 6 Cell structure evolution during simulations calculated with model-B
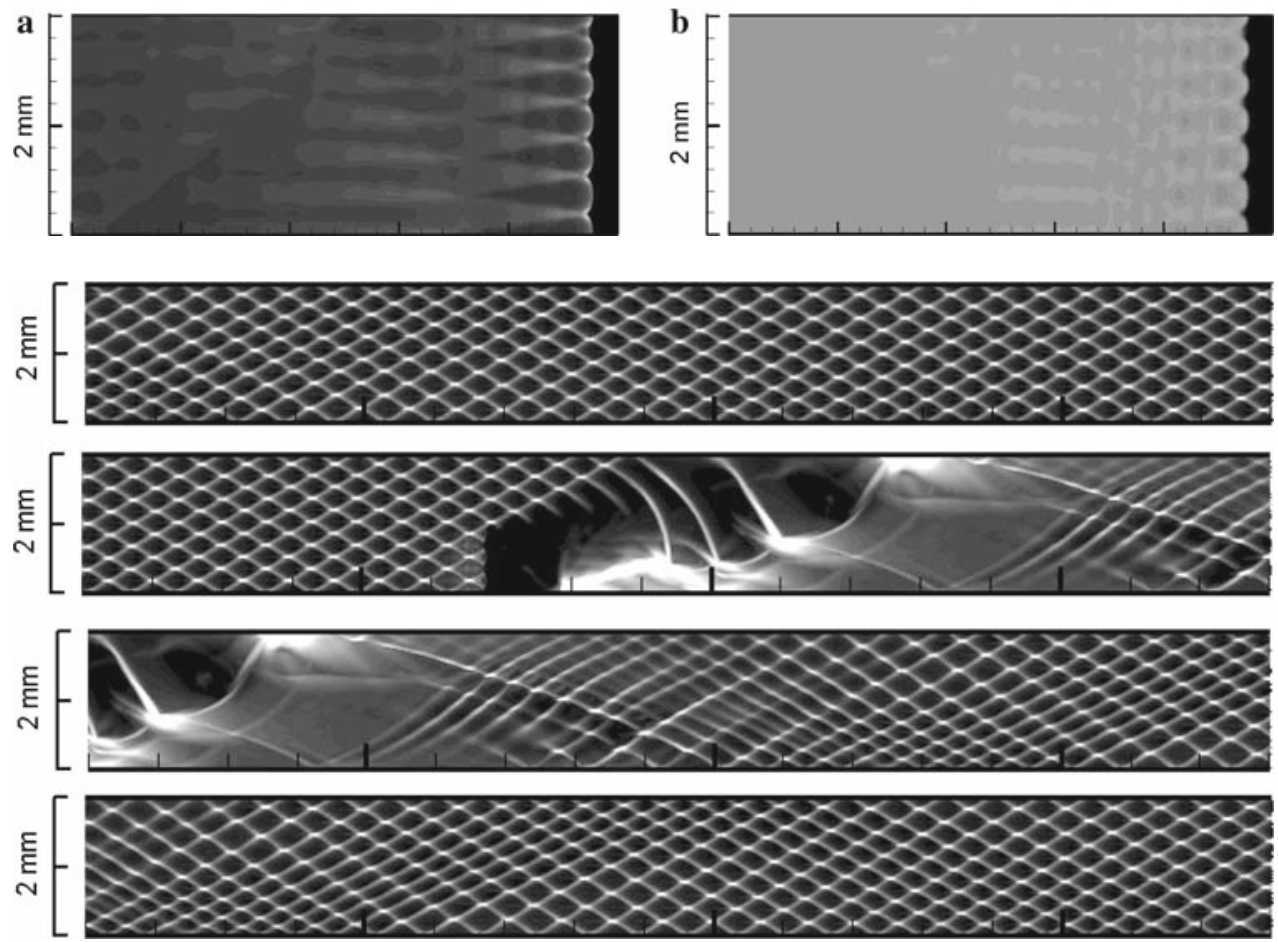

As discussed above in the one-dimensional simulations, the chemical reaction kinetics of model-B is simulated by mis-matching the variable $\gamma(Z)$ and the variable $R(Z)$, so it cannot be used to reproduce correctly the von Neumann spike; therefore, the cellular detonation structures cannot be predicted well in the two-dimensional cases. This problem is treated in Ref. [6] by introducing a grid-dependent preexponential factor $K$ in the Eq. (5), and $K$ has to be tuned according to different grid resolutions to produce the reasonably correct von Neumann spike. The smaller pre-exponential factor $K$ is used for coarse grid, and the larger one for a fine grid.

The key problem arising from the model-B's prediction is induced by the Arrhenius law expressed in the Eq. (5). The Arrhenius law implicates that only molecules which possess the internal energy greater than the activation energy $E_{\mathrm{a}}$ can react, but the activation temperature is applied in its expression, instead of the activation energy. The internal energy is actually a function of temperature and $\gamma$, but the Arrhenius law does not account for the effect of a varying $\gamma$ on the internal energy. At the detonation front, the specific heat ratio $\gamma$ of the gas mixture varies drastically, and imposes a signifi- cant influence on the chemical reaction rate. Therefore, we proposed model-C by introducing the averaged internal energy into the Arrhenius law to replace the activation temperature for calculating the chemical reaction rate.

Figure 7 shows pressure and temperature distributions of two-dimensional cellular detonation simulated with modelC. There is one pair of triple-wave points in this $8 \mathrm{~mm}$ wide channel. The triple-wave structure of the detonation front can be clearly observed, i.e., the Mach stem, the incident shock wave, and the transverse waves. The cellular patterns are shown in Fig. 8 and the cell width is $8.0 \mathrm{~mm}$. This result is the best one among three detonation models, being the closest to the experimental result [11] of $8.4 \mathrm{~mm}$. The ratio of cell width to length is about 0.61 , being in a good agreement with the experimental value of 0.6 [12]. It is also demonstrated in this case that the cellular pattern is mainly dominated by the chemical reaction kinetic properties of detonation models, and is recovered from any flow disturbance placed in front of the detonation front after a period of reshuffle.

The comparison of two-dimensional numerical simulations demonstrated that model-C has the best performance in predicting both thermodynamic parameters and chemical
Fig. 7 Pressure and temperature contours of two-dimensional cellular detonation simulated with model-C. a Pressure; b temperature
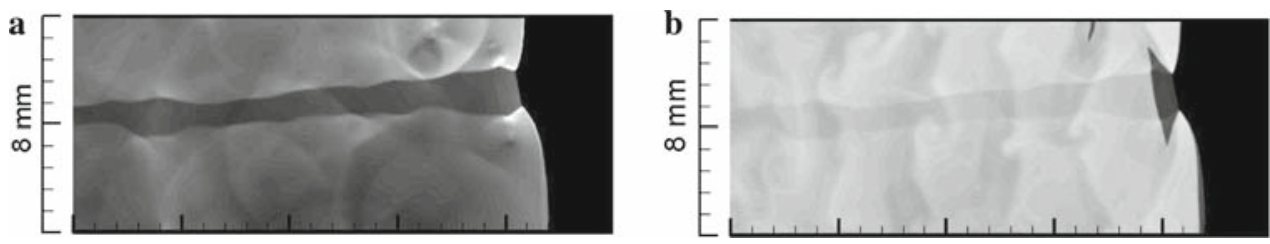
Fig. 8 Cellular cell pattern evolution during simulations calculated with model-C
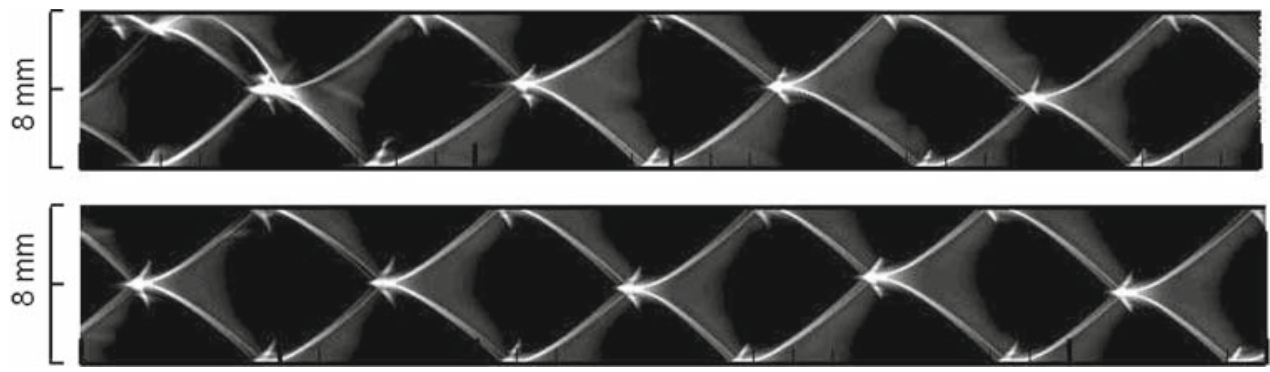

reaction kinetics of detonations among the three examined models. The mechanism understandable is that the specific heat ratio $\gamma$ varies from 1.4 of reactants to 1.24 of products across the detonation front, and the variation of $\gamma$ relative to the reactants is only $11.1 \%$, but results in a $40 \%$ variation of the averaged internal energy calculated by way of $1 /(\gamma-1)$. Therefore, the specific heat ratio $\gamma$ imposes a significant influence on the chemical reaction kinetics, and plays an important role in the Arrhenius law applications.

\section{Conclusions}

In this study, the variable specific heat ratio was demonstrated to impose a significant influence on the Arrhenius law in the overall one-step chemical reaction models. Using activation temperature instead of activation energy in the Arrhenius law results in mis-prediction of detonation because the specific heat ratio and gas constant vary drastically at the detonation front, and the internal energy is not exactly proportional to the absolute temperature. So, the activation energy should be introduced into the Arrhenius law application instead of the activation temperature. On the basis of the conventional overall one-step detonation models, a new overall one-step detonation model was proposed by introducing a variable specific heat ratio and a variable gas constant to cast the averaged internal energy into the Arrhenius law. The numerical experiments of one- and two-dimensional detonation simulations demonstrated that this new model predicts well chemical reaction kinetics of detonations, has better performances among the presently applied overall one-step detonation models, and is capable of capturing the cellular detonation with reasonable accuracy.

\section{References}

1. Kuo, K.K.: Principles of Combustion, p. 115. Wiley, New York (1986)

2. Gamezo, V.N., Desbordes, D., Oran, E.S.: Formation and evolution of two-dimensional cellular detonations. Combustion Flame 116, 154-165 (1999)

3. Sharpe, G.J.: Transverse waves in numerical simulations of cellular detonations. J. Fluid Mech. 447, 31-51 (2001)

4. Ma, F., Choi, J.Y., Yang, V.: Thrust chamber dynamics and propulsive performance of single-tube pulse detonation engines. J. Propulsion Power 21(3), 512-526 (2005)

5. Liu, Y.F., Sato, H., Tsuboi, N., Hayashi, A.K.: Study of the onestep, two-step and full-mechanism detonation model. In: Proceedings of the 43rd Symposium (Japanese) on Combustion, pp. 486487 (2005)

6. Cho, D.R., Won, S.H., Choi, J.Y., Ma, F.H., Yang, V.: Threedimensional unstable detonation wave structures in pipes. AIAA2006-957 (2006)

7. Petersen, E.L., Hanson, R.K.: Reduced kinetics mechanisms for ram accelerator combustion. J. Propulsion Power 15(4), 591600 (1999)

8. Endo, T., Fujiwara, T.: A simplified analysis on a pulse detonation engine mode. Trans. Jpn. Soc. Aeronaut. Space Sci. 44(146), 217$222(2002)$

9. Shu, C.W., Osher, S.: Efficient implementation of essentially nonoscillatory shock-capturing schemes II. J. Comput. Phys. 83, 32 78 (1989)

10. Steger, J.L., Warming, R.F.: Flux vector splitting of the inviscid gasdynamic equations with application to finite-difference methods. J. Comput. Phys. 40, 263-293 (1981)

11. Kaneshige, M., Shepherd, J.E.: Detonation database, Technical Report FM97-8, GALCIT, July 1997. See also the electronic hypertext version at http://www.galcit.caltech.edu/detn_db/html/

12. Edwards, D.H., Thomas, G.O., Nettleton, M.A.: The diffraction of a planar detonation wave at an abrupt area change. J. Fluid Mech. 95(1), 79-96 (1979) 